\title{
Influencia de la edad en la mortalidad de pacientes con insuficiencia cardiaca
}

\author{
F. J. RUIZ-RUIZ, P. SAMPERIZ LEGARRE, F. J. RUIZ LAIGLESIA, J. I. PÉREZ \\ CALVO, J. L. MORALES RULL, R. DOLZ ASPAS, A. FLAMARIQUE PASCUAL, \\ M. AMORES FERRERAS
}

Servicio de Medicina Interna. Hospital Clínico Universitario “Lozano Blesa”. Zaragoza

\author{
INFLUENCE OF AGE IN MORTALITY OF PATIENTS WITH HEART \\ FAILURE
}

\section{RESUMEN}

Objetivo: La insuficiencia cardiaca (IC) representa uno de los motivos de ingreso más frecuentes en los Servicios de Medicina Interna. La elevada edad de los pacientes es una de las principales características de este tipo de ingreso.

Nuestro objetivo fue estudiar la influencia de la edad en la mortalidad, a medio plazo, de la IC tras un episodio de descompensación.

Metodo: Se revisaron las historias clínicas de los pacientes dados de alta, con diagnóstico de IC, durante el periodo comprendido entre septiembre de 2000 y agosto de 2001 , y se registró su situación a fecha 1 de enero de 2003. Un total de 215 pacientes fueron revisados.

Resultados: El número total de defunciones durante el periodo de seguimiento fue de 60 (27,9\% del total de pacientes). Según aumentaba la edad de los pacientes se observó un mayor número de mujeres y un menor empleo de la ecocardiografía y del tratamiento con beta bloqueantes y anticoagulación oral en pacientes con fibrilación auricular. Aplicando el modelo de riesgos proporcionales de Cox, la edad (OR 1,043 IC $95 \%$ 1,002-1,085), la estancia media (OR 1,04, IC 95\% 1,003-1,078), la presencia de diabetes mellitus (OR 2,51, IC 95\% 1,37-4,60) y la ausencia de anticoagulación oral en caso de fibrilación auricular (OR 2,71, IC 95\% 1,10-6,60) fueron factores pronósticos independientes.

Conclusión: La edad constituye un importante factor pronóstico en pacientes con IC. Por ello deberían realizarse estudios clínicos que incluyeran pacientes con estas características, lo que permitiría conocer mejor la evolución de la IC en este grupo de población.

PALABRAS CLAVE: Insuficiencia cardiaca. Pronóstico. Mortalidad. Edad. Medicina Interna. Ancianos.

\begin{abstract}
Objective: Heart failure $(H F)$ is one of the most frequent diagnosis in Internal Medicine Units. High age of patients is an important characteristic in these admissions.

Our objective was to evaluate how age interact with mortality, to medium-term, in HF after a decompensated episode.

Method: Through the patient medical record we obtained data about patients hospitalised in a Internal Medicine Unit, with HF diagnosis, for a year (from September 2000 to August 2001) and their survival at 1st January 2003. 215 patients were reviewed.

Results: During follow-up, 60 patients died (27.9\% of overall). In elderly patients, we observed a higher number of women and less use of echocardiography and treatment with beta-blockers and warfarin (in patients with atrial fibrillation). In Cox proportional-hazards model, age (OR 1.043 IC 95\% 1.002-1.085), days of hospitalization (OR 1.04 IC 95\% 1.003-1.078), diabetes mellitus (OR 2.51 IC 95\% 1.37-4.60) and do not prescribe warfarin in patients with atrial fibrillation (OR 2.71 IC 95\% 1.10-6.60) were independent prognostic factors.

Conclusions: Age was an important prognostic factor in patients with HF. Clinical trials should be done in patients with these characteristics. So, we can know better clinical evolution of HF in this population.
\end{abstract}

KEY WORDS: Heart failure. Prognosis. Mortality. Age. Internal Medicine. Elderly.

Ruiz-Ruiz FJ, Samperiz Legarre P, Ruiz Laiglesia FJ, Pérez Calvo JI, Morales Rull JL, Dolz Aspas R, Flamarique Pascual A, Amores Ferreras M. Influencia de la edad en la mortalidad de pacientes con insuficiencia cardiaca. An Med Interna (Madrid) 2005; 22: 424-428.

\section{INTRODUCCIÓN}

La insuficiencia cardiaca es uno de los motivos de ingreso hospitalario más frecuentes, constituyendo el $5 \%$ del total de hospitalizaciones. El número total de ingresos por IC se estima en casi 80.000 por año (1). Dos de la características mas representativas de este síndrome son la heterogeneidad de los pacientes que la padecen y el mal pronóstico a corto y medio plazo. Datos recientes acerca de las características de estos pacientes en nuestro país indican la existencia de una población con una edad media elevada, por encima de los 77 años y una mortalidad tras un primer episodio de IC del $24 \%$ en el 
primer año, siendo el porcentaje de reingreso tras el primer episodio del $32 \%$ el primer año (2).

Diferentes factores relacionados con el pronóstico de estos pacientes han sido señalados, entre ellos la edad (3-5). Dado que esta es una de las principales características del paciente con IC ingresado en Medicina Interna, decidimos estudiar como influía en la mortalidad a medio plazo.

\section{OBJETIVO}

Evaluar la influencia de la edad en la mortalidad a medio plazo de pacientes dados de alta de un Servicio de Medicina Interna con diagnóstico de insuficiencia cardiaca.

\section{MATERIAL Y MÉTODOS}

Se revisaron los informes de los paciente dados de alta del Servicio de Medicina Interna (unidad B) del Hospital Clínico Universitario de Zaragoza, en el periodo comprendido entre septiembre de 2000 y agosto de 2001, y se seleccionaron aquellos en los que la IC figuraba como diagnóstico, bien principal o secundario. No se seleccionaron los pacientes que fallecieron durante el ingreso.

Se obtuvieron datos acerca de las características del paciente (edad, sexo, clase funcional basal, etc.), diagnóstico previo de IC, enfermedades concomitantes, número de fármacos empleados en el tratamiento, tipo de diagnóstico realizado (clínico o ecocardiográfico) y datos acerca de las recomendaciones dadas al alta y el tratamiento prescrito. Con fecha 1 enero de 2003 se recogió, mediante entrevista telefónica, el estado de los pacientes referido a reingreso y mortalidad.

\section{MÉTODO ESTADÍSTICO}

Las variables cuantitativas se expresaron como la media \pm la desviación estándar. Las variables cualitativas se expresaron en porcentajes. Para la comparación de medias se utilizo el test t-Student y para la comparación de proporciones el test de chi-cuadrado. En el estudio de supervivencia se utilizó el método de Kaplan-Meier para la construcción de curvas y se compararon con el test de log-rank. Para evitar el efecto de variables de confusión o modificadoras del efecto en el estudio de supervivencia se utilizó el modelo de riesgos proporcionales de Cox. Para el análisis de los datos se utilizó el programa estadístico SPSS.

\section{RESULTADOS}

Un total de 215 pacientes fueron seleccionados. Sus características epidemiológicas se recogen en la tabla I. La edad media de los pacientes incluidos en el estudio fue de $77,11 \pm 9,43$ años. Por sexos, la edad media de las mujeres $(78,23 \pm 9,5$ años $)$ fue superior a la de los hombres $(75,47 \pm$ 9,13 años), siendo la diferencia estadísticamente significativa $(\mathrm{p}=0,035)$. Para valorar la influencia de las diferentes edades se dividió la muestra en cuartiles, obteniéndose los grupos que se muestran en la tabla II. Se observó como según aumentaba la edad, aumentaba el porcentaje de muje-
TABLA I

CARACTERÍSTICAS EPIDEMIOLÓGICAS DE LA POBLACIÓN ESTUDIADA

\begin{tabular}{lr}
\hline Variable & $N^{0}$ sujetos $(\%)$ \\
\hline Edad mayor a 75 años & $141(65,6 \%)$ \\
Sexo (Varones) & $87(40,5 \%)$ \\
Antecedentes de IC & $128(59,5 \%)$ \\
NYHA basal & \\
Clase I & $14(6,5 \%)$ \\
Clase II & $31(14,4 \%)$ \\
Clase III & $107(49,8 \%)$ \\
Clase IV & $63(29,3 \%)$ \\
Etiología & \\
Hipertensión arterial & $60(28,03 \%)$ \\
Cardiopatía isquémica & $57(26,63 \%)$ \\
Cardiopatía de origen valvular & $22(10,28 \%)$ \\
Fallo del ventrículo derecho & $33(15,42 \%)$ \\
Precipitante & \\
Infección & \\
Alteración del ritmo cardiaco & $71(33,3 \%)$ \\
Realización de ecocardiografía & $32(15 \%)$ \\
Disfunción sistólica confirmada & $77(35,8 \%)$ \\
Disfunción diastólica confirmada & $33(15,3 \%)$ \\
Fibrilación auricular & $44(20,5 \%)$ \\
Diabetes mellitus & $89(41,1 \%)$ \\
\hline
\end{tabular}

TABLA II

CARACTERÍSTICAS DE LOS DIFERENTES CUARTILES DE EDAD EN LOS QUE FUE DIVIDIDA LA MUESTRA

\begin{tabular}{lcccc}
\hline Cuartil & $N^{\circ}$ Sujetos & Rango (años) & Edad media & DE \\
\hline 1 & 50 & $44-72$ & 64,36 & 7,97 \\
2 & 51 & $73-77$ & 74,82 & 1,32 \\
3 & 60 & $78-83$ & 79,93 & 1,68 \\
4 & 54 & $84-96$ & 87,94 & 2,87 \\
\hline
\end{tabular}

res frente a varones, siendo la diferencia estadísticamente significativa, $\mathrm{p}<0,05$, (Fig. 1). En el grupo de pacientes incluidos en el cuartil 4 el porcentaje de mujeres fue del $72,2 \%$ frente al $27,8 \%$ de varones.

No se evidenció un mayor número de pacientes con antecedente de IC conocida en relación con la edad. En los pacientes en quienes el diagnóstico de IC fue clínico, la edad media fue de 78,74 \pm 8,6 años, mientras que en aquellos en quienes el diagnóstico fue corroborado por ecocardiografía, la edad media fue de 74,19 $\pm 8,6$ años, siendo la diferencia estadísticamente significativa $(\mathrm{p}=0,001)$. Esta tendencia se acrecentaba según aumentaba la edad del paciente, así, en los pacientes incluidos en el primer cuartil se realizó un ecocardiograma en el $46 \%$ de sujetos, mientras que en los 


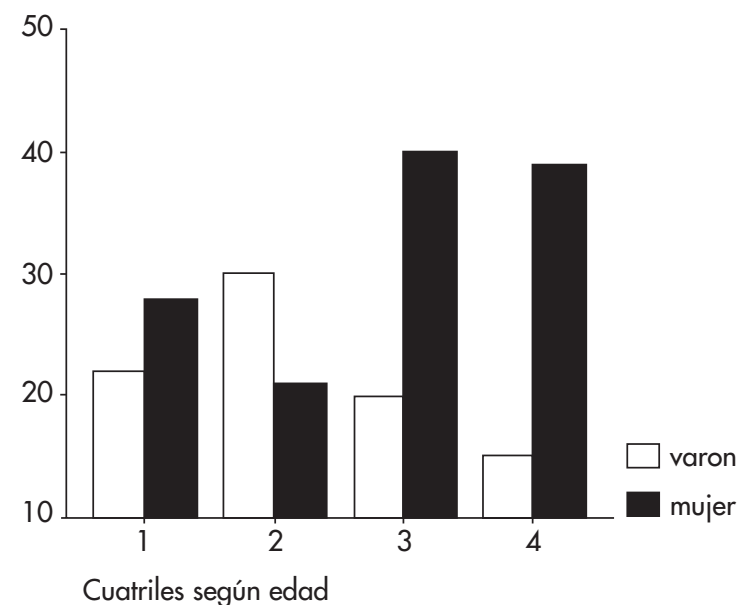

Fig. 1. Composición por sexo de los diferentes cuartiles.

pacientes incluidos en el cuarto cuartil se realizó al 22,2\% del total, siendo $\mathrm{p}=0,03$. No se objetivaron diferencias de edad en función de la clase funcional basal según la NYHA. En cuanto a patología asociada se observó un mayor número de enfermedades concomitantes en pacientes por encima de los 80 años sin que las diferencias fueran significativas. Estudiando la patología asociada en función de la edad solo se objetivaron diferencias significativas en aquellos pacientes que presentaban enfermedad hepática de cualquier tipo. Los pacientes con enfermedad hepática eran mas jóvenes, $66,67 \pm 10,64$ años frente a 77,77 $\pm 9,04$ años, siendo la diferencia estadísticamente significativa $(\mathrm{p}<0,001)$. No se observó relación entre la edad y la presencia de hipertensión arterial, diabetes mellitus, enfermedad pulmonar obstructiva crónica, insuficiencia renal o enfermedades neurológicas de cualquier tipo.

Los pacientes con fibrilación auricular concomitante presentaron una edad ligeramente superior al resto de la muestra, $78,38 \pm 7,47$ años y 76,21 $\pm 10,53$ respectivamente, con una diferencia no estadísticamente significativa $(\mathrm{p}=0,28)$.

Con respecto al tratamiento, la prescripción de IECA no se vio influenciada por la edad. En cambio, se observó una tendencia a prescribir beta bloqueantes y anticoagulantes orales (en los casos en los que el paciente presentaba fibrilación auricular) en pacientes de menor edad. La edad media de los pacientes tratados con beta bloqueantes fue de $71 \pm 14,39$ años y la de los pacientes tratados con anticoagulantes orales de 75,93 \pm 5,72 años. En ninguno de los casos la diferencia fue significativa, siendo $\mathrm{p}=0,09$ y $\mathrm{p}=0,08$, respectivamente. No se observaron diferencias en la prescripción de IECA o beta bloqueantes en los diferentes cuartiles. En cambio, sí se observó una menor prescripción en los pacientes del cuarto cuartil (el 4,5\% de los pacientes de este grupo con fibrilación auricular estaban anticoagulados), comparados con los del primer cuartil de edad (el 62,5\% de los pacientes de este grupo con fibrilación auricular estaban anticoagulados), siendo la diferencia estadísticamente significativa. La estancia media de los pacientes estudiados fue de 10,32 \pm 7,32 días, no viéndose modificada por la edad.
Durante el seguimiento, 100 pacientes $(46,5 \%)$ reingresaron. No se evidenciaron diferencias en el número de reingresos en función de la edad.

El número de defunciones durante el periodo de seguimiento fue de 60 (27,9\% del total de pacientes seleccionados). La edad media de los pacientes fallecidos $(79,43 \pm 9,58$ años $)$ fue superior a la de quienes sobrevivieron (76,21 $\pm 9,24$ años), con una diferencia estadísticamente significativa $(\mathrm{p}=0,024)$.

Analizando la mortalidad a lo largo del periodo de seguimiento se observó una mayor mortalidad en aquellos pacientes de más edad. Dividiendo la muestra en cuartiles según la edad y aplicando el método de Kaplan-Maier se observó una diferencia estadísticamente significativa $(\mathrm{p}=0,01)$, con una menor supervivencia en el grupo de mayor edad (Fig. 2).

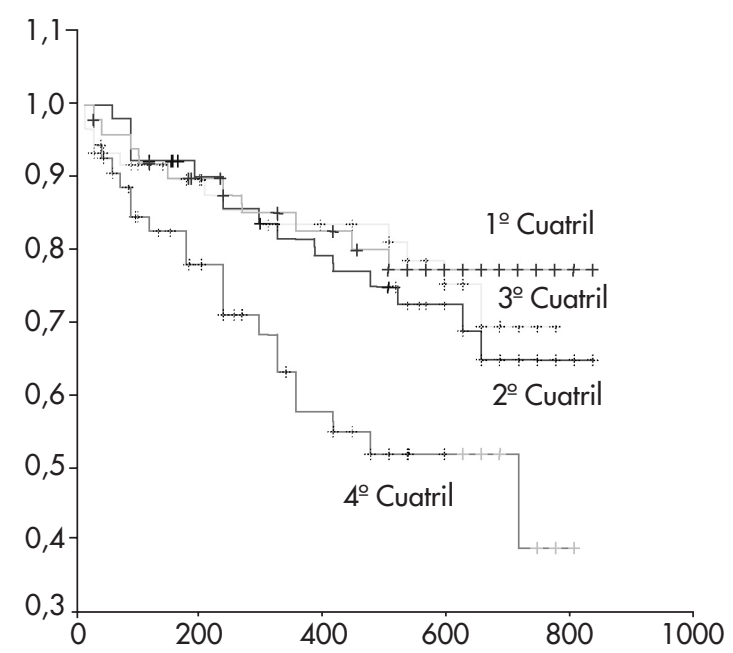

Fig. 2. Curvas de mortalidad, según el método de Kaplan-Meier, para los diferentes cuartiles de edad.

La presencia de diabetes se asoció a una mayor mortalidad, falleciendo a lo largo del seguimiento el 38,7\% de los pacientes diabéticos frente al 23,8\% de pacientes no diabéticos, siendo la diferencia estadísticamente significativa $(\mathrm{p}=0,028)$.

Aplicando el modelo de riesgos proporcionales de Cox para evaluar mortalidad se observó que la edad fue un factor de riesgo independiente con una odds ratio (OR) de 1,043 (IC 95\% 1,002-1,085 $\mathrm{p}=0,042)$. Comparando los pacientes de mayor edad con aquellos pertenecientes al primer cuartil, los primeros presentaban una OR para mortalidad de 2,85 (IC $95 \% 1,13-7,19 \mathrm{p}=0,026)$. Otras variables asociadas a un mayor riesgo de mortalidad fueron la estancia media con una OR de 1,04 (IC 95\% 1,003-1,078 p = 0,032), la coexistencia de diabetes mellitus con una OR de 2,51 (IC 95\% 1,37-4,60 p $=0,003)$ y presentar fibrilación auricular y no estar en tratamiento anticoagulante, siendo en este caso la OR de 2,71 (IC $95 \%$ 1,1-6,6 $\mathrm{p}=0,03)$. Para el resto de variables incluidas en el modelo (tipo de IC, etiología de la IC, IC previa, sexo, NYHA basal, tratamiento con IECA o beta bloqueantes, presencia de fibrilación auricular, EPOC, insuficiencia renal, enfermedad hepática o neurológica) no se estableció un riesgo de mortalidad estadísticamente significativo. 


\section{DISCUSIÓN}

Los resultados obtenidos muestran que la edad es uno de los principales factores pronósticos a medio plazo en pacientes con IC ingresados en un Servicio de Medicina Interna. Las características epidemiológicas de estos pacientes, con una edad elevada y una elevada tasa de comorbilidad asociada, han sido recogidas de manera constantes en los estudios que sobre insuficiencia cardiaca en Medicina Interna se han realizado en nuestro entorno $(6,7)$.

A pesar de que en algunos estudios la edad no se ha catalogado como factor pronóstico en IC, en otros ha demostrado ser un marcador independiente de riesgo para mortalidad y reingreso. Sin embargo, son pocos los estudios que han analizado, de forma específica, la contribución de la edad en el pronóstico de la IC, ya que en la mayoría de estudios la edad actuaba como una variable más a estudio. Junto a la elevada edad, otros factores pronósticos descritos en este grupo de población son el internamiento en residencias geriátricas, una clase funcional basal IV (en la escala de la NYHA), la disfunción ventricular sistólica y la coexistencia de insuficiencia renal (8).

Al igual que en nuestro trabajo, otros autores han constatado como la edad se relaciona con un peor pronóstico y un menor seguimiento de las guías de practica clínica en IC (9). En nuestro estudio, el diagnóstico de la IC mediante ecocardiografía se vio influenciado por la edad. No se realizó ecocardiograma en el $21,7 \%$ de los pacientes sin diagnóstico previo o constatado de IC. Analizando el grupo de pacientes en quienes no se realizó ecocardiografía se observa que estos eran pacientes de más edad y había un mayor número de mujeres. A pesar de que el porcentaje de sujetos en quienes no se empleó esta técnica es similar al descrito en otras series que incluyen pacientes con características similares a las de los pacientes de nuestro estudio, es reflejo de la infrautilización de la ecocardiografía en el diagnóstico de la IC en pacientes ancianos $(10,11)$. En nuestro estudio no se objetivaron una mayor mortalidad en función del tipo de IC. Aunque el escaso porcentaje de pacientes a quienes se practico ecocardiograma no muestra diferencias, estos datos serían consistentes con estudios mas recientes en los que se atribuye el mismo pronóstico a pacientes con disfunción sistólica o diastólica (12). La no realización de ecocardiograma condiciona el infradiagnóstico de la disfunción sistólica y con ello la infrautilización de fármacos que han demostrado su eficacia en este tipo de patología, como los IECA, beta bloqueantes y espironolactona.
Se objetivó un menor empleo de determinados fármacos, en concreto, beta-bloqueantes y anticoagulantes orales. Respecto al tratamiento anticoagulante en la fibrilación auricular no reumática, atendiendo a distintos ensayos clínicos, son los pacientes de mayor edad los que mayor provecho pueden obtener de la anticoagulación en la prevención de enfermedad cerebrovascular cardioembólica (13). Sin embargo el hecho de que muchos de estos pacientes ancianos presenten una limitada calidad de vida o una corta expectativa de vida puede influir en la menor anticoagulación de estos pacientes.

Destaca el bajo porcentaje de pacientes en quienes se prescribió beta bloqueantes, un hecho ya referido por otros autores $(14,15)$. Un mayor empleo de estos fármacos supondría un efecto añadido a la terapia convencional, sin embargo son necesarios estudios que demuestren la seguridad de estos fármacos en pacientes por encima de los 85 años.

No se evidenció una mayor comorbilidad según aumentaba la edad, hecho este que podría ser explicado porque aquellos pacientes con mayor número de enfermedades asociadas tendrían menos posibilidades de envejecer. Entre las enfermedades asociadas, solo la diabetes demostró ser un factor pronóstico independiente. La mayor incidencia de insuficiencia cardiaca en pacientes con diabetes, y el peor pronóstico de estos es un hecho conocido (16). El peor pronóstico de los pacientes diabéticos estaría determinado por la relación entre la diabetes mellitus y la enfermedad cardiovascular y la aterosclerosis (17).

Tras realizar el ajuste estadístico oportuno para evitar el efecto de factores de confusión o modificadores, la edad fue un marcador pronóstico independiente de mortalidad a medio plazo. Estos hallazgos son concordantes con estudios realizados con anterioridad, que muestran una mayor mortalidad en pacientes de mayor edad. Diferentes hipótesis se han dado para explicar este hecho que puede parecer obvio. Así se habla de alteraciones estructurales, funcionales y neurohormonales según aumenta la edad o del tratamiento subóptimo de los pacientes ancianos. Sin embargo, ninguna de estas teorías han demostrado suficiente solidez para explicar el fenómeno $(5,18)$.

En conclusión, la edad es un importante factor pronóstico en los pacientes con insuficiencia cardiaca. Dado el aumento en la prevalencia de este síndrome en los pacientes de mayor edad, que son quienes, además, presentan un peor pronóstico, sería necesaria la realización de un mayor número de estudios en muestras que reúnan las características de los pacientes vistos en los Servicios de Medicina Interna.

\section{Bibliografía}

1. Rodríguez -Artalejo F, Banegas Banegas JR, Guallar-Castillón P. Epidemiología de la insuficiencia cardiaca. Rev Esp Cardiol 2004; 57: 16070 .

2. Grupo de Trabajo en Insuficiencia Cardiaca de la Sociedad Española de Medicina Interna (SEMI). Insuficiencia Cardiaca en los Servicios de Medicina Interna. Med Clin (Barc) 2002; 118: 605-10.

3. McDermott MM, Feinglass J, Lee PI et al. Systolic function, readmission rates, and survival among consecutively hospitalized patients with congestive heart failure. Am Heart J 1997; 134: 728-36.

4. Mosterd A, Cost B, Hoes AW et al. The prognosis of heart failure in the general population. The Rotterdam Study. Eur Heart J 2001; 22: 1318-27.
5. Gustafsson F, Torp-Pedersen C, Seibaek M, Burchardt H, Kober L for the DIAMOND study group. Effect of age on short and long-term mortality in patients admitted to hospital with congestive heart failure. Eur Heart J 2004; 25: 1711-17.

6. Permanyer Miralda G, Soriano N, Brotons C, Moral I, Pinar J, Cascant $\mathrm{P}$, et al. Características basales y determinantes de la evolucion en pacientes ingresados por insuficiencia cardiaca en un hospital general. Rev Esp Cardiol 2002; 55: 571-78.

7. Formica F, Chivite D, Manito N, Osma V, Miravet S, Pujol R. One-year follow-up of heart failure patients after their first admission. QJM 2004; 97: 81-6. 
8. Wang R, Mouliswar M, Denman S, Kleban M. Mortality of the institutionalized old-old hospitalized with congestive heart failure. Arch Intern Med 1998; 158: 2464-8.

9. Pulignano G, Del Sindaco D, Tavazzi L, Lucci D, Gorini M, Leggio F, et al. Clinical features and outcomes of elderly outpatients with Herat failure followed up in hospital cardiology units: data from a large nationwide cardiology database (IN-CHF Registry). Am Heart J 2002; 143: 45-55.

10. Bellotti P, Badano LP, Acquarone N, Grifo R, Lo Pinto G, Maggioni AP et al. Specialty-related differences in the epidemiology, clinical profile, managament and outcome of patients hospitalized for heart failure. The OSCUR study. Eur Heart J 2001; 22: 596-604.

11. Senni M, Rodeheffer RJ, Tribouilloy CM, Evans JM, Jacobsen SJ, Bayley KR et al. Use of echocardiography in the management of congestive heart failure in the community. J Am Coll Cardiol 1999; 33: 164-70.

12. Ojeda S, Anguita M, Muñoz JF, Rodríguez MT, Mesa D, Franco M et al. Características clínicas y pronóstico a medio plazo de la insuficiencia cardiaca con función sistólica conservada. ¿Es diferente de la insuficiencia cardíaca sistólica?. Rev Esp Cardiol 2003; 56: 1050-6.
13. Falk RH. Atrial fibrillation. N Engl J Med 2001; 344: 1067-78.

14. Salas Coronas J, Montiel Trujillo A, López Martínez A, Zambrana García JL, Delgado Fernández M, Diez García F. Factores que intervienen en la no prescripción de betabloqueantes en los pacientes con infarto agudo de miocardio. An Med Interna (Madrid) 2001; 18: 187-90.

15. Ahmed A, Dell'Italia LJ. Use of beta-blockers in older adults with chronic heart failure. Am J Med Sci 2004; 328: 100-11.

16. Croft JB, Giles WH, Pollard RA, Keenan NL, Casper ML, Anda RF. Heart failure survival among older adults in the United States. A poor prognosis for an emerging epidemic in the Medicare population. Arch Intern Med 1999; 159: 505-10.

17. Nichols GA, Gullion CM, Koro CE, Perros SA, Brown JB. The incidence of congestive heart failure in type 2 diabetes: an update. Diabetes Care 2004; 27 : 1879-84.

18. van Veldhuisen DJ, Boomsma F, de Kam PJ, Man in't Veld AJ, Crijns HJ, Hampton JR, et al. Influence of age on neurohormonal activation and prognosis in patients with chronic heart failure. Eur Heart J 1998; 19: 753-60. 